\title{
DETECCIÓN DE LA MUERTE SÚBITA CARDÍACA USANDO EL MÉTODO ESPECTRAL ADAPTATIVO SOBRE LA ONDA T: ESTUDIO EXPERIMENTAL SOBRE BASES DE DATOS PÚBLICAS
}

\author{
DETECTION OF SUDDEN CARDIAC DEATH \\ USING THE ADAPTIVE SPECTRAL METHOD ON \\ THE T WAVE: AN EXPERIMENTAL STUDY ON \\ PUBLIC DATABASES
}

\author{
Marco Flores-Calero $^{1, *, 2}$, Melisa Pilla-Barroso ${ }^{3}$
}

\section{Resumen}

El análisis de la alternancia de la onda T (TWA, T-wave alternants) constituye una de las principales técnicas para determinar la presencia del síndrome de muerte súbita cardíaca (MSC). Entre los métodos existentes para determinar TWA se encuentra el método espectral adaptativo (SM-Adaptativo), el cual utiliza distribuciones en tiempo-frecuencia (TFD, time-frecuency distribution) para el análisis. El objetivo del estudio es aplicar este método sobre las principales bases de datos públicas con el fin de detectar la presencia o ausencia de alternancias y obtener parámetros de calidad del método en mención. El método fue probado en señales sintéticas, 90 señales sin TWA y 450 con TWA; por otro lado, se utilizaron 10 señales de la base de datos TWADB de Physionet pertenecientes a pacientes sanos y 26 señales de pacientes con factores de riesgo asociados a la MSC. En las pruebas con señales sintéticas se obtuvo una sensibilidad de $94,89 \%$, especificidad de $92,22 \%$ y exactitud de 94,44\%. En cuanto a las pruebas en la base de datos el método presenta una exactitud del $80,56 \%$, lo que indica que el método SM-Adaptativo permite detectar TWA con una exactitud aceptable, además, que presenta mayor robustez frente a ruido y a la estacionariedad de datos.

Palabras clave: ECG, muerte súbita cardíaca, alternancia de la onda T, SM-Adaptativo

\section{Abstract}

T-wave alternans (TWA) analysis is one of the main techniques for determining whether an individual is at risk of sudden cardiac death (SCD). Among the existing methods for determining TWA is the adaptive spectral method (SM-Adaptive), which uses time-frequency distributions (TFD) for the analysis. The objective of the study is to apply the method on main public databases in order to detect the presence or absence of alternations, and to obtain quality parameters of the aforementioned method. The method was tested on synthetic signals, 90 signals without TWA and 450 with TWA; on the other hand, 10 signals from Physionet's TWADB database belonging to healthy patients and 26 signals from patients with risk factors associated to SCD were used. Tests with synthetic signals showed a sensitivity of $94.89 \%$, specificity of $92.22 \%$ and accuracy of $94.44 \%$. As for the tests in the database, the method exhibits an accuracy of $80.56 \%$, which indicates that the SM-Adaptive method enables detecting TWA with an acceptable accuracy and, in addition, it shows greater robustness against noise and stationary data.

Keywords: ECG, sudden cardiac death, T-wave alternans, SM-Adaptive.

\footnotetext{
$\overline{1, *}$ Departamento de Eléctrica, Electrónica y Telecomunicaciones, Universidad de las Fuerzas Armadas, Sangolquí, Ecuador. Autor para correspondencia ${ }^{-}$: mjflores@espe.edu.ec. (D) http://orcid.org/0000-0001-7507-3325

${ }^{2}$ Departamento de Sistemas Inteligentes, Tecnología I\&H, Latacunga, Cotopaxi, Ecuador.

${ }^{3}$ Profesional en libre ejercicio, Quito, Ecuador. (D) http://orcid.org/0000-0002-1441-1236
}

Recibido: 06-02-2020, aprobado tras revisión: 21-05-2020

Forma sugerida de citación: Flores-Calero, M. y Pilla-Barroso, M. (2020). «Detección de la muerte súbita cardíaca usando el método espectral adaptativo sobre la onda T: Estudio experimental sobre bases de datos públicas». INGENIUS. N. ${ }^{\circ} 24$, (julio-diciembre). pp. 59-67. DOI: https://doi.org/10.17163/ings.n24.2020.06. 


\section{Introducción}

La muerte súbita cardíaca (MSC) es un evento que provoca de manera rápida e inesperada la muerte de un individuo aparentemente sano, o con una enfermedad cardíaca conocida, pero con poca probabilidad de muerte. La MSC se produce debido a una inestabilidad eléctrica del corazón, lo que impide que se produzca un latido cardíaco y como consecuencia el corazón deja de bombear sangre al resto del cuerpo. En el cerebro se presenta una falta de flujo sanguíneo, lo que provoca la pérdida de oxígeno; esto hace que pierda el conocimiento, de forma abrupta, y se produzca la muerte del individuo.

Entre las principales causas de la MSC están las de origen cardiovascular [1]. Las enfermedades cardíacas que se encuentran más asociadas con la MSC son las siguientes: enfermedad de la arteria coronaria, cardiomiopatías, anomalías electrofisiológicas, cardiopatía valvular y anomalías cardíacas congénitas, entre otras $[2]$.

Asimismo, se estima que la MSC es la responsable de 3 a 7 millones de muertes cada año a nivel mundial [2], [3]. En los países latinoamericanos no se tienen registros oficiales relacionados con la MSC, lo que impide que se pueda conocer de manera precisa su incidencia. Los informes sobre este problema no resultan uniformes, debido a que la MSC supone un problema multifactorial y varía de acuerdo con la edad. Además, existe la posibilidad de que continúe incrementando como consecuencia del aumento de la enfermedad coronaria (tabaquismo, obesidad, diabetes mellitus, hipertensión arterial, aumento del colesterol), convirtiéndole en un importante desafío por resolver.

En la actualidad las terapias más utilizadas para la prevención de la MSC son la medicación y el desfibrilador cardioversor implantable (DCI) [3], sin embargo, al ser pruebas invasivas y de alto costo resulta evidente la búsqueda de técnicas que permitan una rápida detección de este tipo de fenómenos de gran interés en el ámbito social y tecnológico.

El ECG es una de las herramientas más utilizada para el estudio y diagnóstico de enfermedades cardíacas. Permite registrar la actividad eléctrica del corazón al colocar electrodos en la superficie del cuerpo, de modo que se obtiene una secuencia de latidos cardíacos descritos en un conjunto de ondas (P, Q, R, S, T), segmentos y complejos $[4,5]$.

Entre los mecanismos existentes para medir la inestabilidad eléctrica del corazón en el ECG se encuentran la prolongación del QT, dispersión del QT, potenciales tardíos, alternancia de onda T (TWA) y turbulencia de la frecuencia cardíaca [6]. La TWA ha sido utilizada para el análisis de la repolarización ventricular, que se manifiesta en el ECG como fluctuaciones periódicas de la amplitud de la onda $\mathrm{T}$ [7].

La estimación de TWA implica la medición latido a latido, de la variabilidad de la amplitud, duración y forma del segmento ST-T del registro ECG [8]. La señal TWA se encuentra generalmente en el rango de los microvoltios; por lo que, se hace necesario el uso de técnicas avanzadas de procesamiento digital de señales y algoritmos computacionales para su detección.

En los últimos años, se han propuesto varios procedimientos de análisis de TWA. Las técnicas mayormente utilizadas han sido desarrolladas en el dominio temporal, entre ellas está el método de la media móvil (MMA), el cual calcula el valor de TWA utilizando la diferencia del valor medio de los latidos pares e impares [9]; otro método es el de correlación (CM), que detecta alternancias utilizando la correlación cruzada [10]. Entre los métodos desarrollados en el dominio de la frecuencia se encuentran el método espectral (SM), el cual emplea la transformada discreta de Fourier (DFT) para analizar la componente de frecuencia a 0,5 ciclos por latido [11]. Un método similar es el de la demodulación compleja (CD), el cual ajusta una señal sinusoidal a la frecuencia de 0,5 ciclos por latido de las ondas $\mathrm{T}$ alineadas [12]. La principal desventaja de estos métodos es que son muy sensibles a las señales con ruido. Últimamente las técnicas de inteligencia artificial están siendo utilizadas para la clasificación de MSC [5].

Con el fin de superar las limitaciones de los métodos antes descritos Ghoraani et al. [13] proponen un método de detección y cuantificación de TWA llamado SM-Adaptativo, el cual toma como base el proceso del método SM y utilizando distribuciones de tiempofrecuencia hace el análisis de TWA; de modo que permite representar las variaciones espectrales de TWA a lo largo del tiempo, a la vez que cuenta con la capacidad de seguir estructura no estacionarios. Por otro lado, utiliza la factorización de matrices no negativas (NMF) con el fin de separar el ruido de la señal TWA, y de este modo mejorar la capacidad de detección.

En este trabajo el principal objetivo es evaluar el desempeño del método SM-Adaptativo en distintos escenarios. Para lograr esto se han utilizado un conjunto de señales sintéticas (simulaciones) con y sin TWA agregado con amplitudes variables, y ante distintos niveles de ruido. El primer grupo, es decir, las señales sintéticas con TWA agregado se generaron a partir de cinco señales base a las cuales se les ha sumado alternancias con valores distintos de amplitud: $10 \mu \mathrm{V}$, $20 \mu V, 50 \mu V, 100 \mu V$ y $200 \mu V$. De este modo se tienen 25 señales; las alternantes se generan a partir de tres formas de onda: gaussiana, triangular y rectangular, lo que da lugar a 75 señales. Finalmente, se agregaron cinco niveles diferentes de ruido blanco gaussiano a todas las señales ECG, SNR: 10, 20, 30, 40 y 50. Estas señales junto a las señales libres de ruido dieron un total de 450 señales de prueba. Para el segundo grupo, conformado por señales sintéticas con TWA, se tomaron cinco señales base, con tres formas 
de onda distintas (15 señales), y cinco niveles de ruido. Obteniendo junto con las señales libres de ruido 90 . De este modo entre los dos grupos se tienen un total 540 señales sintéticas de prueba. A continuación, se desarrolló la prueba en señales reales para lo cual se aplicó el método SM-Adaptativo a 36 señales de la base de datos TWADB de Physionet. Las señales pertenecen a pacientes sanos y a pacientes que presentan factores de riesgo de sufrir MSC. Se hizo el cálculo de la magnitud de TWA con el fin de determinar la existencia o por el contrario la ausencia de alternancias de la señal de prueba. Con lo que se pudo establecer los valores de sensibilidad, especificidad y exactitud. Además, se hizo una comparación de dichos parámetros con los métodos SM y MMA que se encuentran disponibles en el software TWAnalyser [14].

\section{Materiales y métodos}

El presente trabajo se centra en el análisis de señales cardíacas; 540 señales sintéticas y 36 señales de la base de datos TWADB, con el fin de detectar TWA en las señales utilizando el método SM-Adaptativo, y a continuación, obtener los parámetros de calidad del algoritmo, para evaluar el desempeño.

Inicialmente el método requiere de una etapa de preprocesamiento para filtrar las señales y para detectar y segmentar los puntos característicos de la señal ECG.

A continuación, se aplica el método SM-Adaptativo seguido de la técnica NMF (factorización de matrices no negativas) con el fin de detectar la presencia o ausencia de alternancias de la señal. Esto hace posible la clasificación de las señales con y sin TWA. La Figura 1 muestra las etapas de la metodología propuesta.

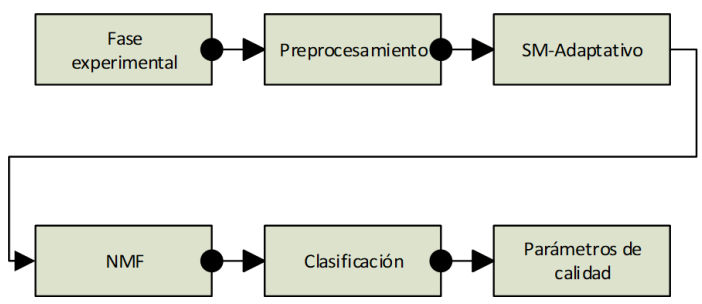

Figura 1. Metodología propuesta para la detección de MSC.

Con este procedimiento se pueden obtener los parámetros de calidad del método; sensibilidad, especificidad y exactitud.

\subsection{Desarrollo de la fase experimental}

En primer lugar, se probó el desempeño del algoritmo en señales sintéticas, 450 señales sintéticas que representan a señales sin TWA, con diferentes niveles de ruido y amplitud de la alternancia variable. Además, un segundo grupo de 90 señales sin TWA agregado, es decir, amplitud $0 \mu \mathrm{V}$ y con diferentes valores de ruido.

Luego, se seleccionaron 36 señales tomadas de la base de datos T-Wave Alternans Database (TWADB) presentada en [15], la cual incluye señales pertenecientes a pacientes sanos y a pacientes que presentan factores de riesgo de MSC. Los registros ECG han sido muestreados a $500 \mathrm{~Hz}$ con una duración aproximada de dos minutos.

Las 36 señales se encuentran divididas de la siguiente forma, 10 señales corresponden a pacientes sanos, y las 26 señales restantes a pacientes con factores de riesgo de MSC, estos registros se han obtenido de pacientes con infartos de miocardio, isquemia transitoria, taquiarritmias ventriculares entre otros.

\subsection{Preprocesamiento}

Las señales cardíacas, generalmente, presentan ruido, que perturba y distorsiona la información contenida en la señal. El ruido es causado por interferencias externas, como el movimiento del cuerpo, la respiración y contracciones musculares, mala colocación de los electrodos, etc. Es por ello por lo que es necesario una etapa de preprocesamiento robusto, que incluya la eliminación del ruido, la extracción de interferencias en distintas frecuencias externas y la corrección de la desviación de la línea de base [4].

Los métodos utilizados deben garantizar que se realiza un filtrado adecuado, a la vez que, no se pierde información relevante, ya que las alternancias, al encontrarse a nivel de microvoltios suele confundirse con componentes de ruido. En este caso se persigue mantener las características alternantes, y a la vez, eliminar el ruido.

Por otro lado, como prerrequisito para el funcionamiento del método SM-Adaptativo es necesario extraer el segmento ST-T, de cada latido. Para lo cual, es necesario un método de extracción de características y uno de segmentación de las distintas ondas, segmentos y complejos que componen la señal ECG [5].

\subsection{Descripción de SM-Adaptativo}

El método SM-Adaptativo utiliza distribuciones en tiempo-frecuencia para obtener una representación de las componentes de frecuencia de la señal a lo largo del tiempo, se encuentra compuesto por dos etapas, i) alineación y ii) estimación del TFD adaptativo.

\subsubsection{Alineación}

Con las ondas alineadas se construye una matriz A, como se muestra en la Ecuación (1), de dimensiones $\mathrm{M} \times \mathrm{N}$, donde $\mathrm{M}$ es el número de latidos, en este caso 128, y N es la longitud del segmento ST-T. 
$A=\left[\begin{array}{cccc}T_{1}(1) & T_{1}(2) & \cdots & T_{1}(N) \\ T_{2}(1) & T_{2}(2) & \cdots & T_{2}(N) \\ T_{3}(1) & T_{3}(2) & \cdots & T_{3}(N) \\ \vdots & \vdots & \cdots & \vdots \\ T_{M}(1) & T_{M}(2) & \cdots & T_{M}(N)\end{array}\right]=\left[\begin{array}{llll}A_{1} & A_{2} & \cdots & A_{N}\end{array}\right]$

Las filas representan los segmentos ST-T de cada latido y las columnas describen las variaciones latido a latido del segmento ST-T. La representación gráfica de la matriz de alineación A se muestra en la Figura 2.
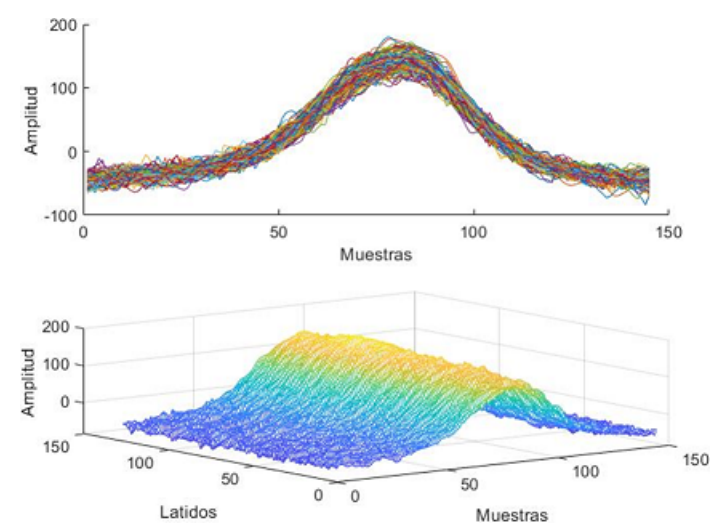

Figura 2. Segmentos ST-T alineados (128 segmentos) con una longitud de 132 muestras, de la señal TWADB55

\subsubsection{TFD adaptativo.}

TFD es una representación bidimensional de la energía de una señal en términos del tiempo y la frecuencia. El método TFD adaptativo utiliza el algoritmo Matching Pursuit para descomponer una señal $\mathrm{x}$ en átomos de tiempo-frecuencia. Una vez que la señal se descompone se utiliza la distribución de Wigner-Ville para obtener la distribución de energía en el dominio de tiempo-frecuencia, usando la Ecuación (2).

$$
V(t, f)=\sum_{i=1}^{I}\left|\alpha_{\gamma i}\right|^{2} W V G_{\gamma i}(t, f)
$$

Aplicando el método TFD Adaptativo a la matriz A se genera la matriz Vi y calculando el promedio se construye una matriz definida por la Ecuación (3).

$$
V_{\frac{M}{2} \times M}=\frac{1}{N} \sum_{i=1}^{N} V_{i}
$$

La magnitud de TWA se calcula tomando los valores de energía a 0,5 ciclos por latido. Para estimar el valor de TWA se requiere la energía a 0,5 ciclos por latido $\mathrm{T}(\mathrm{t})$ y los valores de energía del ruido presente en el intervalo 0,36 a 0,49 ciclos por latido.

Las Figuras 3 y 4 muestran una representación gráfica del TFD adaptativo sobre dos señales cardíacas de la base de datos TWADB [15].

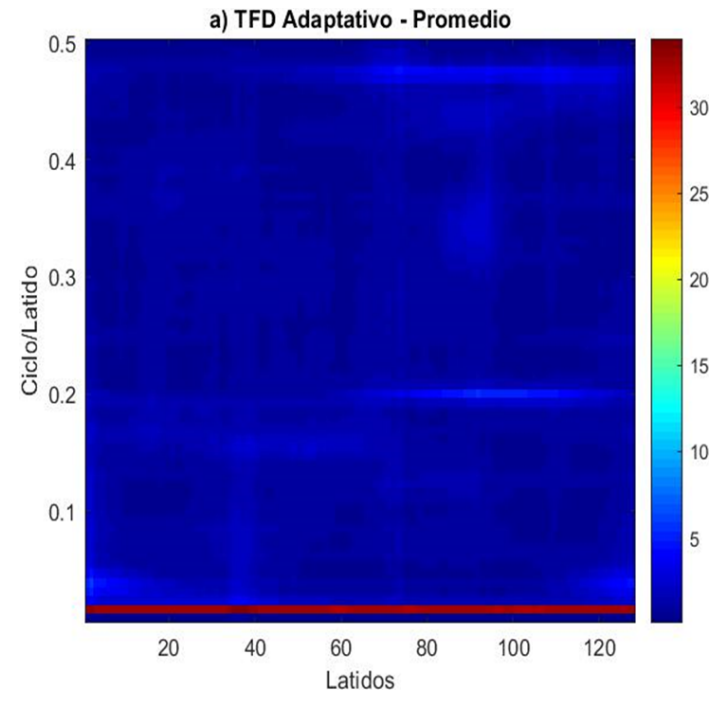

Figura 3. TFD adaptativo promedio de la señal TWADB55 correspondiente a una señal de un paciente sano; a $0,5 \mathrm{cpl}$ se observa valores bajo de alternancia

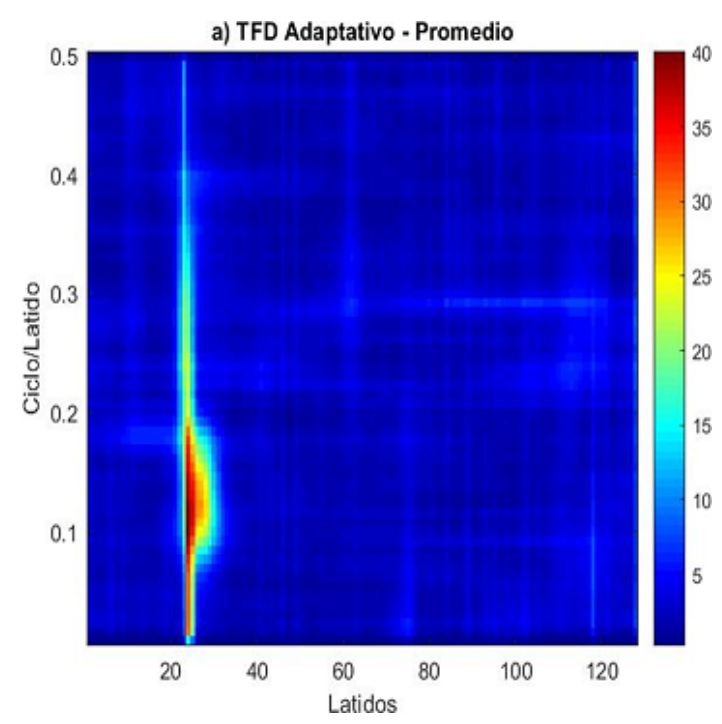

Figura 4. TFD adaptativo promedio de la señal TWADB12 correspondiente a una señal de un paciente con factores de riesgo de MSC; a $0.5 \mathrm{cpl}$ se observa valores bajo de alternancia, sin embargo, entre las muestras $20 \mathrm{y}$ 40 se tiene un posible componente alternante

\subsection{Factorización de matrices no negativas}

En esta etapa se construye una nueva matriz $V_{l \times M^{\prime}}$, en este caso $I=16$ y $M$ es igual a la longitud de la ventana de análisis, en este caso 128. NMF factoriza la matriz de entrada $V$ en dos matrices de menor tamaño $W_{m \times r}$ y $H_{r \times n}$. Tomando un valor de $r=3$, se consiguen tres vectores, $W_{1}, W_{2}$ y $W_{3}$. Los componentes representativos de la magnitud de TWA se agrupan en un solo vector al que se lo representa como $W_{t}$. 
La magnitud de TWA se expresa de acuerdo con la Ecuación (4) y, de este modo, se consigue separar los componentes alternantes de los componentes de ruido.

$$
T W A=w_{t} h_{t}
$$

Finalmente, se calcula el valor de TWA del vector $w_{t}$ que es el componente con la mayor magnitud TWA, de acuerdo con la Ecuación (5).

$$
f=\operatorname{Real} \sqrt{T-\mu_{\text {ruido }}}
$$

Las Figuras 5 y 6 muestran la separación de los componentes alternantes del ruido. La gráfica superior indica los componentes alternantes, en la parte inferior se muestra el ruido extraído de la señal.
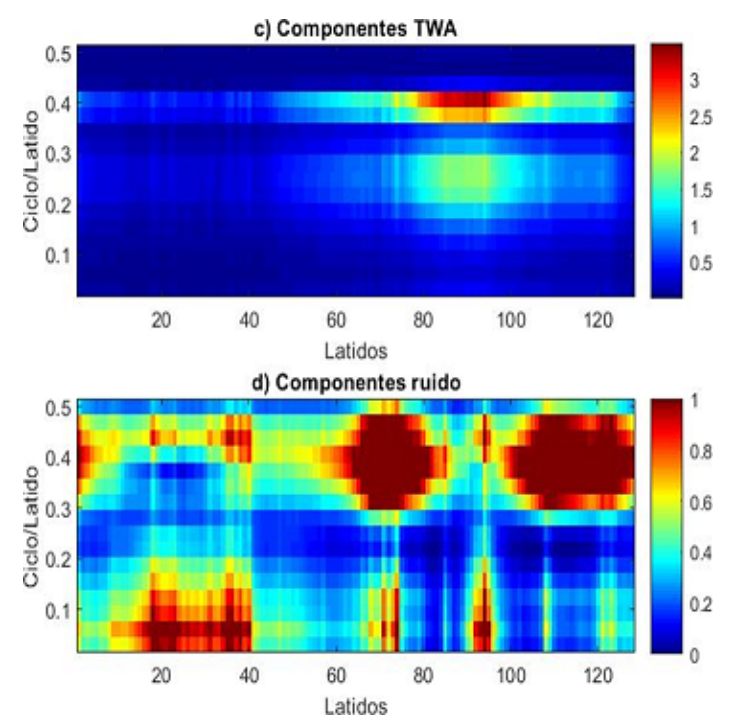

Figura 5. Descomposición de la señal TWADB55 en sus componentes alternantes y ruido
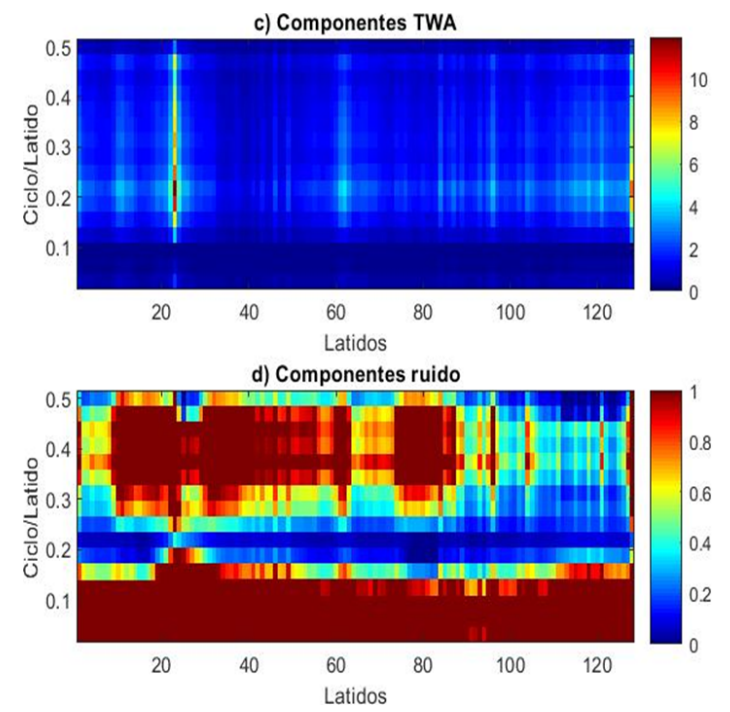

Figura 6. Descomposición de la señal TWADB12 en sus componentes alternantes y ruido
Las Figuras 7 y 8 corresponden a la señal factorizada en tres componentes; el componente con la mayor magnitud de TWA se indica en la gráfica.

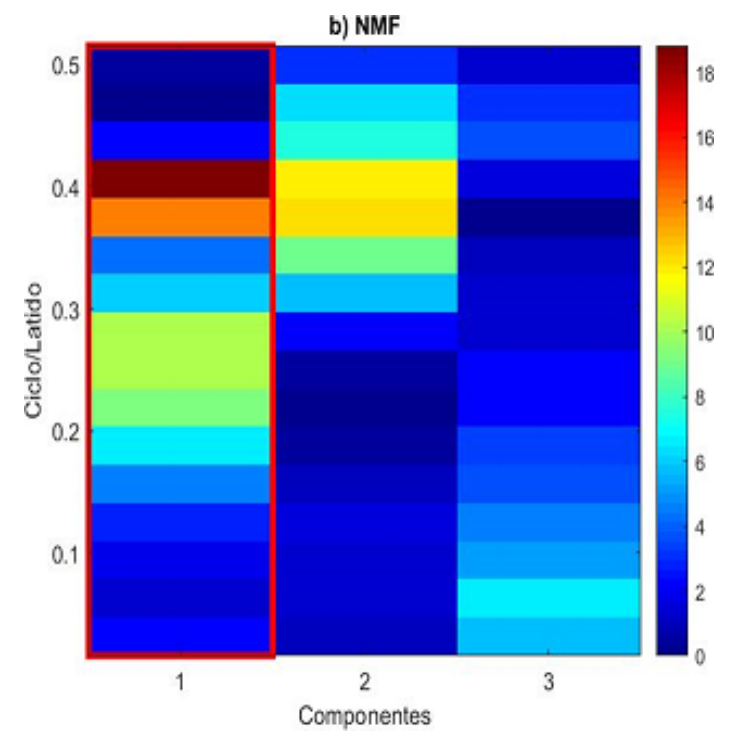

Figura 7. Componente con la mayor magnitud de TWA de la señal TWADB55, a 0,5 bcpl se hace el cálculo de TWA

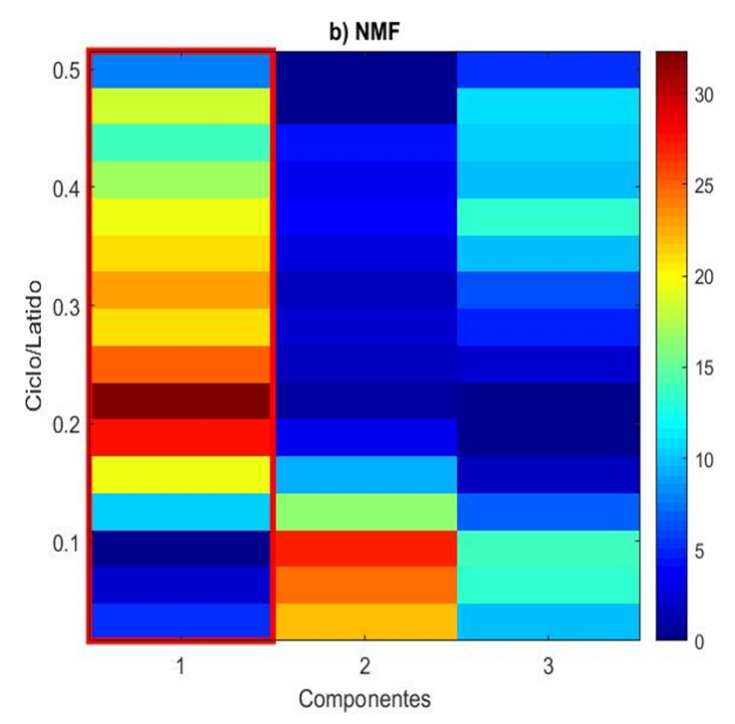

Figura 8. Componente con la mayor magnitud de TWA de la señal TWADB12, a $0.5 \mathrm{cpl}$ se hace el cálculo de TWA

\subsection{Clasificación}

Inmediatamente, después de calcular la magnitud de TWA, se establece un valor de umbral (Th) de $1,5 \mu V$. Entonces las señales se clasifican de acuerdo con la hipótesis dada por la Ecuación (6).

$$
\begin{aligned}
& f>T h \Rightarrow H_{0} \\
& f<T h \Rightarrow H_{1}
\end{aligned}
$$


Donde $H_{0}$ indica presencia de TWA en la señal, e inversamente, $H_{1}$ indica la ausencia de TWA.

\section{Resultados y discusión}

\subsection{Pruebas con señales sintéticas}

Esta primera prueba fue realizada con el fin de evaluar el comportamiento del método SM-Adaptativo en señales sintéticas, que simulan señales cardíacas, a las que se les ha agregado alternancias artificiales y ruido, en distintos niveles.

El esquema de la Figura 9, muestra las señales sintéticas utilizadas, divididas en dos grupos, y el proceso necesario hasta conseguir los parámetros de calidad.

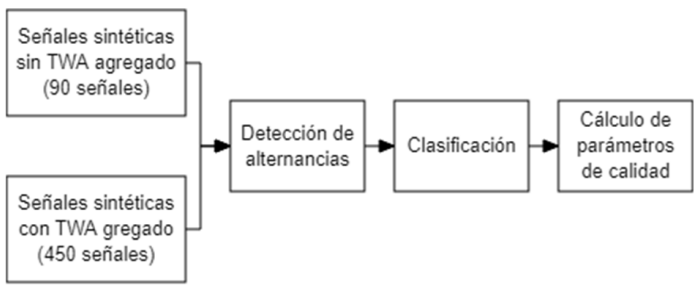

Figura 9. Proceso para la obtención de los parámetros de calidad utilizando señales sintéticas con y sin TWA agregado

Inicialmente, el método SM-Adaptativo fue evaluado en 90 señales sintéticas sin TWA, es decir, señales que simulan señales cardíacas sin presencia de alternancias, las mismas que corresponden a individuos sanos. Los resultados de la clasificación obtenida, al aplicar el método a las 90 señales se encuentra en la Tabla 1, los valores se presentan de acuerdo con los distintos niveles de ruido.

Tabla 1. Clasificación de las señales sintéticas sin TWA de acuerdo con los niveles de ruido agregado

\begin{tabular}{ccc}
\hline SNR (dB) & VN & FP \\
\hline Sin ruido & $15(100 \%)$ & $0(0 \%)$ \\
50 & $15(100 \%)$ & $0(0 \%)$ \\
40 & $15(100 \%)$ & $0(0 \%)$ \\
30 & $15(100 \%)$ & $0(0 \%)$ \\
20 & $12(80 \%)$ & $3(20 \%)$ \\
10 & $11(73,33 \%)$ & $17(26,67 \%)$ \\
\hline Total & $\mathbf{8 3 ( \mathbf { 9 2 , 2 2 } \% )}$ & $\mathbf{2 3 ( \mathbf { 7 , 7 8 } \% )}$ \\
\hline
\end{tabular}

Como resultado se tiene 83 verdaderos negativos (VN) y 7 falsos positivos (FP). Los verdaderos negativos indican, en este caso, que una señal sin TWA ha sido clasificada efectivamente como una señal sin TWA, por otro lado, los falsos positivos indican que una señal sin TWA ha sido clasificada como una señal que sí tiene TWA.

La Tabla 1 muestra que las señales sin presencia de ningún tipo de ruido, que no sucede en señales reales, han sido clasificadas correctamente. De igual forma sucede con las señales con valores altos de SNR, 50, 40 y 30 que se traducen en bajos niveles de ruido (amplitud del ruido), al ser inversamente proporcionales. La clasificación de las señales empieza a generar falsos resultados ante valores de SNR más bajos, para un SNR de 20 y 10 se observa que el número de falsos positivos es de 3 y 17 respectivamente, es decir, para este la clasificación no cuenta con un porcentaje de acierto de $100 \%$ y en todo caso se ha reducido hasta $73,33 \%$ en las señales con el valor más bajo de SNR. Esto indica que el algoritmo es sensible ante altos niveles de ruido (SNR bajo), particularmente a valores de SNR inferiores a 20.

Sin embargo, al calcular la especificidad del método que resulta ser de 92,22\%, el método SM-Adaptativo ha sido capaz de clasificar adecuadamente las señales sin TWA (individuos sanos) incluso ante altos niveles de ruido.

Continuando con las pruebas en señales sintéticas, se han tomado 450 señales sintéticas con TWA artificial agregado, señales que representan a individuos con factores de riesgo asociados a la MSC.

Los resultados de la clasificación se muestran en las Tablas 2 y 3 . La Tabla 2 ha sido tabulada de modo que los resultados se dividen de acuerdo con el nivel de ruido, mientras que la Tabla 3 muestra los resultados de acuerdo con la amplitud de las alternancias agregadas.

Tabla 2. Clasificación de las señales sintéticas con TWA de acuerdo con los niveles de ruido agregado

\begin{tabular}{|c|c|c|}
\hline $\operatorname{SNR}(d B)$ & VP & FN \\
\hline Sin ruido & $75(100 \%)$ & $0(0 \%)$ \\
\hline 50 & $75(100 \%)$ & $0(0 \%)$ \\
\hline 40 & $75(100 \%)$ & $0(0 \%)$ \\
\hline 30 & $74(98.66 \%)$ & $1(1,34 \%)$ \\
\hline 20 & $70(93.33 \%)$ & $5(6,67 \%)$ \\
\hline 10 & $58(77,33 \%)$ & $17(22,67 \%)$ \\
\hline Total & $427(94,89 \%)$ & $23(7,78 \%)$ \\
\hline
\end{tabular}

Tabla 3. Clasificación de las señales sintéticas con TWA de acuerdo con la amplitud de las alternancias agregadas

\begin{tabular}{ccc}
\hline Amplitud $(\mu \mathbf{V})$ & VP & FN \\
\hline 10 & $76(84,44 \%)$ & $14(15,56 \%)$ \\
20 & $85(94,44 \%)$ & $5(5,56 \%)$ \\
50 & $87(96,67 \%)$ & $3(3,33 \%)$ \\
100 & $89(98,89 \%)$ & $1(1,11 \%)$ \\
200 & $90(100 \%)$ & $0(0 \%)$ \\
\hline Total & $\mathbf{4 2 7}(\mathbf{9 4 , 8 9 \% )}$ & $\mathbf{2 3 ( 5 , 1 1 \% )}$ \\
\hline
\end{tabular}

Como resultado de la clasificación realizada por el método SM-Adaptativo, se tienen 427 pruebas diagnos- 
ticadas como verdaderos positivos y 23 falsos negativos. Los verdaderos positivos indican que una señal con TWA ha sido clasificada como tal, por el contrario, los falsos negativos indican que una señal con TWA ha sido clasificada como una señal sin TWA.

En la Tabla 2 se encuentran las respuestas del método frente a señales con diferentes niveles de ruido. Una señal libre de ruido presenta un diagnóstico $100 \%$ correcto, lo que sucede de manera similar con los valores SNR de 50 y 40, es decir, mientras mayor sea el valor del SNR contenido en la señal el método presenta resultados más precisos. Para los valores SNR de $30 \mathrm{~dB}, 20 \mathrm{~dB}$ y $10 \mathrm{~dB}$, la clasificación empieza a presentar clasificaciones erróneas, tal es el caso que para estos valores de SNR se tienen 1, 5 y 17 falsos negativos, respectivamente. Por tanto, el método resulta más sensible a valores de SNR inferiores a $30 \mathrm{~dB}$, dando paso falsas detecciones.

La Tabla 3 muestra la clasificación resultante frente a diferentes valores de alternancia, para una amplitud de $200 \mu \mathrm{V}$ la detección se realiza con $100 \%$ de exactitud, lo que indica que mientras mayor sea la alternancia contenida en la señal resulta más fácil para el algoritmo la detección de las alternancias. Mientras la amplitud de las alternancias va disminuyendo también lo hace el porcentaje de detección, que para $10 \mu \mathrm{V}$ ha decrecido hasta $84,44 \%$, es decir, el método presenta dificultades en la detección cuando la amplitud de la onda alternante es muy pequeña, lo que podría deberse a que se está confundiendo con ruido u otro tipo de interferencia.

Conociendo los verdaderos positivos y falsos negativos de las pruebas, se calculó el valor de sensibilidad que es de 94,89\% un valor aceptable, lo que nos dice que el método cuenta con una alta probabilidad de clasificar correctamente una señal con TWA (individuos con riesgo de MSC). Por lo tanto, el método ha sido capaz de realizar una clasificación correcta con una precisión de 94,44\%, lo que indica que tiene una alta probabilidad de diagnosticar sin errores señales con y sin TWA, con diferentes niveles de ruido y alternancias variables.

\subsection{Pruebas con señales de la base de datos TWADB}

Una segunda forma de evaluación del desempeño de SM-Adaptativo se hizo utilizando señales reales de la base de datos TWADB de Physioet. Las señales seleccionadas corresponden tanto a individuos sanos como a individuos con riesgo de MSC como se muestra en el esquema de la Figura 10.

Las pruebas se realizaron en dos grupos de análisis, por un lado, se tomaron diez señales de pacientes sanos, es decir, señales que no contienen alternancias. Una vez aplicado el método SM-Adaptativo a estas señales, ha dado como resultado la clasificación que se muestra en la Tabla 4, donde se tiene la siguiente información: nombre de la señal escogida, el valor calculado de la alternancia detectada y el diagnóstico generado, verdadero negativo o falso positivo.

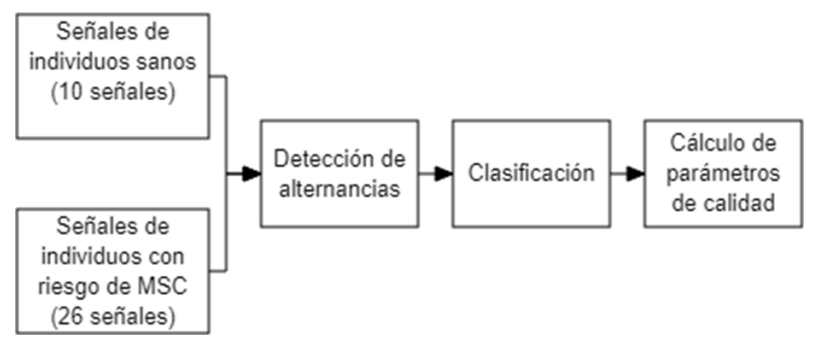

Figura 10. Proceso para la obtención de los parámetros de calidad utilizando señales de la base de datos TWADB

Tabla 4. Resultados generados por el método SMAdaptativo en señales de individuos sanos

\begin{tabular}{ccc}
\hline Señal & Valor TWA & Diagnóstico \\
\hline twa39 & 0 & VN \\
twa46 & 8,3949 & $\mathrm{FP}$ \\
twa55 & 0 & $\mathrm{VN}$ \\
twa60 & 0 & $\mathrm{VN}$ \\
\hline twa10 & 0 & $\mathrm{VN}$ \\
twa23 & 0 & $\mathrm{VN}$ \\
twa61 & 0 & $\mathrm{VN}$ \\
twa62 & 0 & $\mathrm{VN}$ \\
twa71 & 0 & $\mathrm{VN}$ \\
twa93 & 27,1057 & $\mathrm{FP}$ \\
\hline
\end{tabular}

El método clasificó a 8 de las 10 señales como señales sin TWA, es decir, se tienen 8 verdaderos negativos y 2 falsos positivos. Esto indica que el método es capaz de detectar correctamente una señal sin TWA y, por tanto, un individuo sano (especificidad), con un $80 \%$ de probabilidad.

El otro grupo de análisis está conformado por 26 señales correspondientes a pacientes con riesgo de MSC. Los resultados se muestran en la Tabla 5, donde se tiene la siguiente información, nombre de las señales, valor calculado de TWA, en $\mu \mathrm{V}$, y el diagnóstico de la detección.

En el caso de las señales con factores de riesgo de MSC, de las 26 señales, 5 fueron clasificadas como señales sin TWA, es decir, se tienen 5 falsos negativos; por otro lado, las 21 señales restantes han sido clasificadas correctamente y, por tanto, se tiene 21 verdaderos negativos. Con el número de verdaderos positivos y falsos negativos se ha calculado la sensibilidad del método, que es de 80,76\%, es decir, cuenta con una alta probabilidad para detectar correctamente señales sin TWA e individuos con riesgo de MSC.

De acuerdo con los datos de las Tablas 4 y 5 , se tienen 29 señales clasificadas correctamente de un total 
de 36 señales, entre señales con y sin TWA, lo que resulta en que el método cuente con una precisión de $80,56 \%$, es decir, el método tiene una alta probabilidad de detectar y clasificar correctamente las señales con y sin TWA.

Tabla 5. Resultados generados por el método SMAdaptativo en señales de pacientes con factores de riesgo de MSC

\begin{tabular}{ccc}
\hline Señal & Valor TWA & Diagnóstico \\
\hline twa07 & 8,1842 & $\mathrm{VP}$ \\
twa32 & 8,0292 & $\mathrm{VP}$ \\
twa85 & 0 & $\mathrm{FN}$ \\
twa92 & 3,6081 & $\mathrm{VP}$ \\
\hline twa00 & 0 & $\mathrm{FN}$ \\
twa08 & 0 & $\mathrm{FN}$ \\
twa45 & 40,2243 & $\mathrm{VP}$ \\
twa63 & 1,8172 & $\mathrm{VP}$ \\
twa68 & 4,1085 & $\mathrm{VP}$ \\
twa95 & 6,5078 & $\mathrm{VP}$ \\
\hline twa12 & 0 & $\mathrm{FN}$ \\
twa27 & 0 & $\mathrm{FN}$ \\
\hline twa03 & 24,1159 & $\mathrm{VP}$ \\
twa11 & 9,8338 & $\mathrm{VP}$ \\
twa18 & 6,7598 & $\mathrm{VP}$ \\
twa19 & 6,1028 & $\mathrm{VP}$ \\
twa20 & 0 & $\mathrm{FN}$ \\
twa31 & 10,3374 & $\mathrm{VP}$ \\
twa36 & 6,6947 & $\mathrm{VP}$ \\
twa40 & 0 & $\mathrm{FN}$ \\
twa41 & 5,6962 & $\mathrm{VP}$ \\
twa48 & 8,1214 & $\mathrm{VP}$ \\
twa49 & 4,0385 & $\mathrm{VP}$ \\
twa53 & 2,6401 & $\mathrm{VP}$ \\
twa54 & 53,9866 & $\mathrm{VP}$ \\
twa83 & 9,1466 & $\mathrm{VP}$ \\
\hline & &
\end{tabular}

Posteriormente, en este trabajo se hizo una comparación entre el método SM-Adaptativo y los métodos SM y MMA, estos últimos se encuentran implementados en el software TWAnalyser [14].

Para este experimento se tomaron 36 señales descritas anteriormente. Los resultados de especificidad, sensibilidad y precisión que se muestran en la Tabla 6 .

El método MMA es el que presenta más problemas pues al tener una sensibilidad de $100 \%$ y especificidad de $0 \%$, sugiere que clasifica a todas las señales como señales con TWA, aunque no contengan alternancias. Este método toma entonces, a cualquier tipo de perturbación en la señal como una alternancia, lo que es erróneo, es la razón de que el método clasifique equivocadamente al $50 \%$ de las señales de prueba. Por otro lado, el método SM muestra un comportamiento más equilibrado, sin embargo, al compararlo con el método
SM-Adaptativo el porcentaje de precisión en la clasificación es considerablemente inferior, apenas con una precisión de 63,89\%. SM-Adaptativo resulta como un método más aceptable con un comportamiento equilibrado en la detección de señales con y sin TWA.

Tabla 6. Parámetros de calidad de los métodos, SMAdaptativo, SM y MMA

\begin{tabular}{cccc}
\hline Parámetro & SM-Adaptativo & SM & MMA \\
\hline Sensibilidad & $80,76 \%$ & $61,53 \%$ & $100 \%$ \\
Especificidad & $80 \%$ & $70 \%$ & $0 \%$ \\
Precisión & $80,56 \%$ & $63,89 \%$ & $50 \%$ \\
\hline
\end{tabular}

$\mathrm{Al}$ observar los resultados, en los dos tipos de pruebas, es decir, sobre señales sintéticas y registros de la base de datos TWADB, estos indican un desempeño superior del método SM-Adaptativo, respecto a los otros dos métodos, en todos los índices de calidad. En específico, el algoritmo SM-Adaptativo se caracteriza por su robustez en la detección de las alternancias.

\section{Conclusiones}

Los extensivos experimentos mostraron que el método SM-Adaptativo es capaz de detectar alternancias y clasificar las señales correctamente. Adicionalmente se pudo comparar, mediante métricas estándar, el desempeño del algoritmo al ser sometido a diferentes niveles de ruido y niveles de alternancias, presentando ventajas frente a los clásicos SM y MMA. Esta superioridad se debe a que SM-Adaptativo utiliza distribuciones de tiempo-frecuencia que permiten un análisis más detallado de la señal, evitando la pérdida de información relevante contenida en la señal cardiaca y, por lo tanto, se tienen mejores resultados en la detección de alternancias.

En conclusión se puede indicar que SM-Adaptativo es una técnica prometedora para la detección, temprana y no invasiva de la MSC.

En un trabajo futuro se estudiará la capacidad del algoritmo en el análisis de larga duración y su desempeño en sistemas de monitorización móvil destinados a eHealth; todo esto acompañado con el criterio de un experto en cardiología especializado en MSC.

\section{Agradecimientos}

Agradecemos a la Universdad de las Fuerzas Armadas por el financiamiento indirecto a través del proyecto de investigación «EcuSCD-Algoritmos avanzados de aprendizaje máquina para la detección de la muerte súbita cardíaca basado en el método espectral a partir de señales $E C G »$. 


\section{Referencias}

[1] M. Vargas Sanabria, J. C. Vega Chaves, G. Hernández Romero, and G. Montero Solano, "Síndrome de brugada como casusa de muerte súbita de origen cardíaco," Medicina Legal de Costa Rica, vol. 34, pp. 157-164, 03 2017. [Online]. Available: https://bit.ly/2ZEI4Sf

[2] R. Devi, H. K. Tyagi, and D. Kumar, "Early stage prediction of sudden cardiac death," in 2017 International Conference on Wireless Communications, Signal Processing and Networking (WiSPNET), 2017, pp. 2005-2008. [Online]. Available: https: //doi.org/10.1109/WiSPNET.2017.8300112

[3] J. T. Willerson, J. N. Cohn, H. J. J. Wellens, and D. R. Holmes, Cardiovascular Medicine, ser. SpringerLink : Bücher. Springer London, 2007. [Online]. Available: https://bit.ly/3eag8cD

[4] U. Satija, B. Ramkumar, and M. S. Manikandan, "Automated ecg noise detection and classification system for unsupervised healthcare monitoring," IEEE Journal of Biomedical and Health Informatics, vol. 22, no. 3, pp. $722-732,2018$. [Online]. Available: https://doi.org/10.1109/JBHI.2017.2686436

[5] F. Panjaitan, S. Nurmaini, M. Akbar, A. H. Mirza, H. Syaputra, T. B. Kurniawan, and R. Umi $\mathrm{P}$, "Identification of classification method for sudden cardiac death: A review," in 2019 International Conference on Electrical Engineering and Computer Science (ICECOS), 2019, pp. 93-97. [Online]. Available: https: //doi.org/10.1109/ICECOS47637.2019.8984465

[6] E. V. Garcia, C. A. Pastore, N. Samesima, and H. G. Pereira Filho, "T-wave alternans: desempenho clínico, limitações, metodologias de análise," Arquivos Brasileiros de Cardiologia, vol. 96, pp. e53-e61, 03 2011. [Online]. Available: http://dx. doi.org/10.1590/S0066-782X2011005000018

[7] Y. Karplyuk, K. Ivanko, and N. Ivanushkina, "Peculiarities of $\mathrm{t}$ wave alternans detection and evaluation," in 2015 IEEE 35 th International Conference on Electron$i c s$ and Nanotechnology (ELNANO), 2015, pp. 356-361. [Online]. Available: https: //doi.org/10.1109/ELNANO.2015.7146909
[8] O. Karnaukh, Y. Karplyuk, and N. Nikitiuk, "Evaluation of machine learning techniques for ecg t-wave alternans," in 2018 IEEE 38th International Conference on Electronics and Nanotechnology (ELNANO), 2018, pp. 346-350. [Online]. Available: https: //doi.org/10.1109/ELNANO.2018.8477528

[9] G. M. Nijm, S. Swiryn, A. C. Larson, and A. V. Sahakian, "Estimation of t-wave alternans from multi-lead ecg signals using a modified moving average method," in 2008 Computers in Cardiology, 2008, pp. 517-520. [Online]. Available: https://doi.org/10.1109/CIC.2008.4749092

[10] S. Ayub, G. Gupta, and Y. Kumar, "Heart rate calculation and detection of t-wave alternans by correlation method," in 2016 8th International Conference on Computational Intelligence and Communication Networks (CICN), 2016, pp. 200-203. [Online]. Available: https://doi.org/10.1109/CICN.2016.45

[11] T. W. Shen and Y. T. Tsao, "An improved spectral method of detecting and quantifying t-wave alternans for scd risk evaluation," in 2008 Computers in Cardiology, 2008, pp. 609-612. [Online]. Available: https://doi.org/10.1109/CIC.2008.4749115

[12] B. D. Nearing, A. H. Huang, and R. L. Verrier, "Dynamic tracking of cardiac vulnerability by complex demodulation of the t wave," Science, vol. 252, no. 5004, pp. 437-440, 1991. [Online]. Available: http://doi.org/10.1126/science.2017682

[13] B. Ghoraani, S. Krishnan, R. J. Selvaraj, and V. S. Chauhan, "T wave alternans evaluation using adaptive time-frequency signal analysis and non-negative matrix factorization," Medical Engineering \& Physics, vol. 33, no. 6, pp. 700-711, 2011. [Online]. Available: https: //doi.org/10.1016/j.medengphy.2011.01.007

[14] A. Khaustov, S. Nemati, and G. D. Clifford, "TWAnalyser - AT-wave alternans detector," Computers in Cardiology, 2008. [Online]. Available: https://bit.ly/36nUz5L

[15] G. B. Moody, "The physionet/computers in cardiology challenge 2008: T-wave alternans," in 2008 Computers in Cardiology, 2008, pp. 505-508. [Online]. Available: https://doi.org/10.1109/CIC.2008.4749089 